\section{QuantiFERON-TB Test for Annual Screening of Healthcare Workers: Not Yet Ready for Prime Time in Low-Prevalence Countries}

To the Editor-We read with great interest the recently published article by Gandra et $\mathrm{al}^{1}$ that questions the effectiveness of the QuantiFERON-TB Gold In-Tube (QFT-GIT) test (Cellestis) as a screening tool to detect latent tuberculosis infection (LTBI) for healthcare workers (HCWs). We agree with their conclusion that QFT-GIT is not yet completely ready to replace the tuberculin skin test (TST) for screening HCWs in low tuberculosis-incidence countries, considering the high number of positive test results and high reversion rates on repeat testing.

Our practical experience ${ }^{2}$ with the QFT-GIT test showed similar results and presented challenges very similar to those mentioned in the study by Gandra et al. ${ }^{1}$ Annual screening of HCWs for LTBI with TST was the standard infection control practice in our institution (Central Arkansas Veterans Healthcare System, Little Rock, AR) for many years. In November 2008, our hospital replaced the TST with the QFTGIT test for employee testing as well as for screening patients for tuberculosis infection and/or disease. We were confronted with an unexpectedly high number of new converters (more than 20-fold higher than baseline), which led to the dilemma in clinical decision making of whether to offer LTBI treatment since all of these new QFT-GIT converters had a negative TST history. In addition, we encountered high reversion rates (40\%) on repeat QFT-GIT testing, similar to the study by Gandra et al, ${ }^{1}$ and support the concern about poor shortterm reproducibility of QFT-GIT results in serial testing.

Gandra et $\mathrm{al}^{1}$ raised in their discussion the question of using a higher cutoff value for a positive QFT-GIT test result, given the low prevalence of tuberculosis in the United States. We disagree, however, with raising the cutoff values for a positive test result, for many reasons. There is an overlap among the initial interferon- $\gamma$ values in HCWs who reverted to negative status and those who retained positive status in our study as well as in the study by Gandra et al. ${ }^{1}$ Without a gold standard for the diagnosis of LTBI, it is difficult to differentiate false positive QFT-GIT results from true positive results. To address this problem we suggest adopting a borderline zone between IFN- $\gamma$ values of 0.35 and $2.0 \mathrm{IU} / \mathrm{mL}$ and cautious clinical interpretation of values in this range. Repeat testing with QFT-GIT should be considered for HCWs whose IFN- $\gamma$ values are in the "borderline" range and whose TST status is negative.

However, we do agree completely with Gandra et $\mathrm{al}^{1}$ that there is a major clinical learning curve ahead to fully un- derstand the QFT-GIT test characteristics in low tuberculosisprevalence populations. More studies need to be performed to know and understand characteristics of QFT-GIT as a screening tool in this population with a low prevalence of tuberculosis.

We hope that our large study and practical experience with QFT-GIT in the real world, along with the study by Gandra et al, ${ }^{1}$ will help many healthcare organizations that are facing the same challenges that we encountered when TST was replaced by QFT-GIT for annual screening for LTBI. It will also guide the institutions who are in the process of implementing QFT-GIT testing.

\section{ACKNOWLEDGMENTS}

Potential conflicts of interest. All authors report no conflicts of interest relevant to this article.

\section{Manish Joshi, MD, FCCP; ${ }^{1}$ Thomas Monson, $\mathrm{MD}^{2}$ Gail Woods, $\mathbf{M D}^{3}$}

Affiliations: 1. Pulmonary and Critical Care Medicine, Central Arkansas Veterans Healthcare System, Little Rock, Arkansas; 2. Infection Control, Central Arkansas Veterans Healthcare System, Little Rock, Arkansas; 3. Pathology and Lab Medicine, Central Arkansas Veterans Healthcare System, Little Rock, Arkansas.

Address correspondence to Manish Joshi, MD, Central Arkansas Veterans Healthcare System, 4300 West 7th Street, 5C 144, Little Rock, AR 72205 (mjoshi@uams.edu).

Infect Control Hosp Epidemiol 2011;32(5):518-518

(C) 2011 by The Society for Healthcare Epidemiology of America. All rights reserved. 0899-823X/2011/3205-0018\$15.00. DOI: $10.1086 / 659956$

\section{REFERENCES}

1. Gandra S, Scott WS, Somaraju V, Wang H, Wilton S, Feigenbaum M. Questionable effectiveness of the QuantiFERON-TB Gold Test (Cellestis) as a screening tool in healthcare workers. Infect Control Hosp Epidemiol 2010;31:1279-1285.

2. Joshi M, Monson T, Woods G. Practical experience with the QFTGIT assay for LTBI annual testing among US health-care workers in a large tertiary setting. Chest 2010;138:746A.

\section{Reply to Joshi et al}

To the Editor-We are pleased that Joshi et al' report similar outcomes in their recent study ${ }^{2}$ validating our findings. ${ }^{3}$ This strengthens the evidence that the QuantiFERON Gold InTube test (QFT-GIT; Cellestis) is not yet ready for screening healthcare workers (HCWs) for latent tuberculosis infection (LTBI) in the United States.

Coming to the proposal of the borderline zone, we are in 